\title{
Strain measurement in heteroepitaxial layers-Silicon on sapphire
}

\author{
Thad Vreeland, Jr. \\ Division of Engineering and Applied Science, California Institute of Technology, Pasadena, California \\ 91125
}

(Received 5 May 1986; accepted 12 August 1986)

\begin{abstract}
An x-ray diffraction technique is presented for the determination of the strain tensor in an epitaxial layer grown on a crystallographically distinct substrate. The technique utilizes different diffracting planes in the layer and in a reference crystal fixed to the layer, and is illustrated by application to an $\sim 4000 \AA$ (001) silicon layer grown on a (01 $\overline{1} 2)$ sapphire wafer. The principal strains were measured, and the measured strain normal to the layer was found to agree with the normal strain calculated from the measured in-plane strains within the experimental uncertainty of strain measurement. The principal stresses in the plane of the silicon film, calculated from the measured strains were $-0.92 \pm 0.16 \mathrm{GPa}$ in the [100] direction and $-0.98 \pm 0.17 \mathrm{GPa}$ in the [010] direction.
\end{abstract}

\section{INTRODUCTION}

Stresses in epitaxial silicon layers ( $1-46 \mu \mathrm{m}$ thick) grown on (0001) sapphire disks (127-508 $\mu \mathrm{m}$ thick) were deduced by Dumin ${ }^{1}$ from measurements of the curvature of the disk. He reported compressive stresses of the order of $0.1-1 \mathrm{GPa}$ in the silicon layers and found no variation of stress with direction in the plane of the layer.

Calculations of the stresses in silicon layers, strained by thermal contraction of the sapphire as the layer is cooled to ambient temperature, were made by Hughes. ${ }^{2}$ For the case of (001) silicon on (0112) sapphire, and a change in temperature of $1100{ }^{\circ} \mathrm{C}$, principal stresses of -0.87 and $-0.95 \mathrm{GPa}$ were found in the silicon [100] and [010] directions (parallel to sapphire [2110] and [0111] directions), respectively, when the initial stress in the silicon was assumed to be zero. The difference is due to the anisotropic thermal expansion behavior of the sapphire.

Under optimum conditions $\mathrm{x}$-ray diffraction is capable of detecting strains in single crystal silicon of less than $10^{-8}$ (see Ref. 3). Short wavelengths and highorder reflections in a nondispersive $(+,-)$ setting are used in order to achieve such high strain sensitivity. Changes in strain cause directly proportional changes in the Bragg angles for $\mathrm{x}$-ray diffraction from crystal planes, as shown below. The strain sensitivity depends upon the constant of proportionality and the minimum shift in Bragg angle, which can be detected. This minimum detectable shift depends upon the width of the Bragg peak. Thin epitaxial layers have relatively broad Bragg peaks, and the strain sensitivity of the x-ray technique is reduced for thin layers. Crystal defects in the layer also broaden the Bragg peaks and further reduce the strain sensitivity. A strain sensitivity of $\sim 10^{-5}$ is typical for crystal layers a few thousand angstroms thick when using a double crystal diffractometer (with a near- ly perfect first crystal) and sample Bragg angles near $45^{\circ}$. The $x$-ray method of strain measurement has the advantage that it is nondestructive, and it does not require the layer to be on a thin substrate (a thick substrate relative to the layer is optimum since the strain in the substrate is then essentially zero).

This paper presents a method for applying the $\mathrm{x}$-ray technique to measure the strains in a thin heteroepitaxial layer, where the substrate crystal is not useful as a reference for Bragg peak shifts. A stress-free reference crystal is affixed to the surface of the strained film, and the film strains relative to the reference crystal are measured. As an example, a (001) silicon crystal is used on a (001) silicon epialyer grown on $(01 \overline{1} 2)$ sapphire.

\section{EXPERIMENTAL METHOD}

The $\mathrm{x}$-ray rocking curves (reflecting power versus incident angle) are obtained from $(h k l)$ planes of the reference crystal and the strained crystal layer. The difference in angle between Bragg peaks from the reference and the layer is due to two effects: strain in the layer and misorientation between the reference crystal and the layer. These effects are readily separated by using two rocking curves, obtained by reversing the diffraction vectors, as shown by Bonse. ${ }^{4}$ The difference in peak shift of the two rocking curves is twice that due to the strain alone, and the sum is due to strain and the misorientation. A difficulty is encountered in this method when one of the incident diffraction vectors is near the surface normal of a thin epilayer. In this case, the intensity of diffraction from the thin layer may be too weak for accurate strain determination. This condition frequently occurs for one or more of the incident asymmetric diffraction vectors, which must be used to obtain all components of the strain tensor.

An alternative to reversing the diffraction vectors is to use different asymmetric diffracting planes for which 
each of the incident $x$ rays makes a small angle with the layer surface. In the example that follows, we use $\{333\}$ and $\{044\}$ reflections from a surface whose nominal orientation is (001). The Bragg angle shifts are related to strains normal to the layer [determined from two symmetric (004) rocking curves taken with diffraction vectors reversed and incident angles of $\sim 45^{\circ}$ ], and the strains in the plane of the layer are obtained from the asymmetric pairs of reflections. The angle of $x$-ray incidence with respect to the surface in the asymmetric reflections was less than $24^{\circ}$ and gave a Bragg reflection of useable intensity. Reversing the diffraction vectors gave angles of incidence near $120^{\circ}$, and the asymmetric Bragg reflection from the thin epilayer was too weak to be useful for strain measurement using a conventional $x$-ray tube source.

\section{RELATIONSHIP BETWEEN BRAGG ANGLE SHIFT AND STRAIN}

The Bragg angle shift between layer and reference crystals is due to a misorientation angle between the layer and reference and the change in spacing of the Bragg planes in the layer (relative to those in the reference crystal). The change in plane spacing is related to the strains in the layer: the normal strain perpendicular to the layer, $\epsilon_{3}$ (perpendicular strain), and the normal strain parallel to the layer, $\epsilon_{P}$ (parallel strain), in the direction defined by the intersection of the diffraction plane with the layer. The surface of the layer is free of tractions so there is no shear strain in the plane of these two normal strains, and the perpendicular strain is a principal strain. Using peak shifts obtained for three reflections from different planes of the same zone permits calculation of the two strains and the misorientation angle. Peak shifts obtained for two reflections from two additional zones permits determination of the complete strain tensor. When the parallel strains determined for peak shifts using two different zones are principal strains, three rocking curves from planes of one zone and two rocking curves from planes of the other zone permit determination of the three principal strains.

The relationships between the peak shifts and the strains are simplified for the case where one of the diffracting planes $\left(h_{0} k_{0} l_{0}\right)$ is nearly parallel to the layer surface, and has a plane of symmetry perpendicular to it. Two additional diffracting planes of the $[u v w]$ zone, which is the intersection of $\left(h_{0} k_{0} l_{0}\right)$ and the plane of symmetry, are used. We assume that $[u v w]$ lies in the surface and define $\psi_{1}$ as the angle between $\left(h_{0} k_{0} l_{0}\right)$ and the layer surface and $\psi_{0}$ as the angle between $\left(h_{0} k_{0} l_{0}\right)$ and the plane $(h k l)$, as well as its symmetric equivalent $\left(h_{c} k_{c} l_{c}\right)$ in the $[u v w]$ zone. The crystal and incident beam geometry are shown schematically in Fig. 1. The

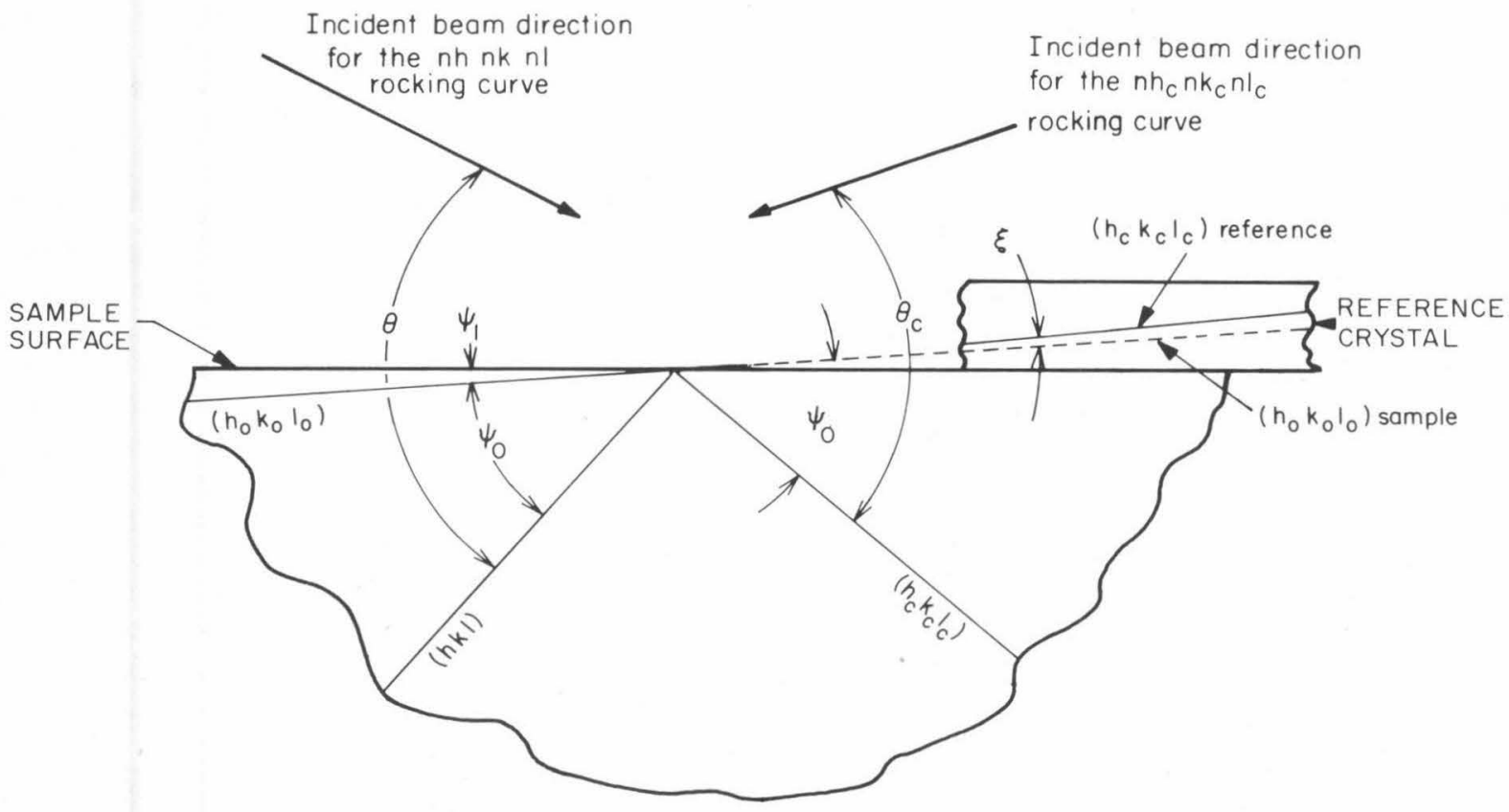

FIG. 1. Schematic drawing of the sample with a plane $\left(h_{0} k_{0} l_{0}\right)$, which makes a small angle $\psi_{1}$ with respect to the surface. Equivalent planes $(h k l)$ and $\left(h_{c} k_{c} l_{c}\right)$ are oriented at $\psi_{0}$ from $\left(h_{0} k_{0} l_{0}\right)$. A reference crystal, shown here on the surface of the sample, is misoriented from the sample by the angular component $\xi$ in the plane of the figure (the diffraction plane). An x-ray rocking curve for the $n h n k n l$ reflection is obtained by scanning $\theta$ about the Bragg peaks from the sample and reference crystals, and a rocking curve for the $n h_{c} n k_{c} n l_{c}$ reflection is obtianed by scanning $\theta_{c}$ about the Bragg peaks. 
Bragg angle shift for each of the equivalent planes and a strain-free reference crystal with a component of misorientation, $\xi$ about $[u v w]$, is

$$
\begin{aligned}
& -\Delta \theta=k_{1} \epsilon_{3}+k_{2} \epsilon_{P}+\xi, \\
& -\Delta \theta_{c}=k_{1 c} \epsilon_{3}+k_{2 c} \epsilon_{P}-\xi,
\end{aligned}
$$

where the subscript $c$ refers to the symmetric equivalent plane and

$$
\begin{aligned}
k_{1}= & \cos ^{2}\left(\psi_{0}+\psi_{1}\right) \tan \theta_{\mathrm{B}} \\
& +\sin \left(\psi_{0}+\psi_{1}\right) \cos \left(\psi_{0}+\psi_{1}\right), \\
k_{2}= & \sin ^{2}\left(\psi_{0}+\psi_{1}\right) \tan \theta_{\mathrm{B}} \\
& -\sin \left(\psi_{0}+\psi_{1}\right) \cos \left(\psi_{0}+\psi_{1}\right), \\
k_{1 c}= & \cos ^{2}\left(\psi_{0}-\psi_{1}\right) \tan \theta_{\mathrm{B}} \\
& +\sin \left(\psi_{0}-\psi_{1}\right) \cos \left(\psi_{0}-\psi_{1}\right), \\
k_{2 c}= & \sin ^{2}\left(\psi_{0}-\psi_{1}\right) \tan \theta_{\mathrm{B}} \\
& -\sin \left(\psi_{0}-\psi_{1}\right) \cos \left(\psi_{0}-\psi_{1}\right), \\
\theta_{\mathrm{B}}= & \text { Bragg angle. } .^{4,5}
\end{aligned}
$$

The sign of the $\xi$ term reverses in Eqs. (1) and (2) as in the case where the diffraction vectors are reversed. ${ }^{4}$ The first two terms in Eqs. (1)-(6) are obtained from consideration of how the perpendicular and parallel strains change the spacing of the Bragg planes [first term in Eqs. (3)-(6) ], and cause them to rotate [second term in Eqs. (3)-(6) $].{ }^{5}$ Taking the sum and difference of Eqs. (1) and (2) we obtain

$$
\begin{aligned}
& \left.(\Delta \theta)+\Delta \theta_{c}\right) / 2=K_{1} \epsilon_{3}+K_{2} \epsilon_{P}, \\
& \left(\Delta \theta-\Delta \theta_{c}\right) / 2=K_{3} \epsilon_{3}+K_{4} \epsilon_{P}+\xi,
\end{aligned}
$$

where

$$
\begin{aligned}
K_{1}=\{ & \tan \theta_{\mathrm{B}}\left[\cos ^{2}\left(\psi_{0}+\psi_{1}\right)+\cos ^{2}\left(\psi_{0}-\psi_{1}\right)\right] \\
& +\sin \left(\psi_{0}+\psi_{1}\right) \cos \left(\psi_{0}+\psi_{1}\right) \\
& \left.+\sin \left(\psi_{0}-\psi_{1}\right) \cos \left(\psi_{0}-\psi_{1}\right)\right\} / 2
\end{aligned}
$$

$$
\begin{aligned}
K_{2}=\{ & \tan \theta_{\mathrm{B}}\left[\sin ^{2}\left(\psi_{0}+\psi_{1}\right)+\sin ^{2}\left(\psi_{0}-\psi_{1}\right)\right] \\
& -\sin \left(\psi_{0}+\psi_{1}\right) \cos \left(\psi_{0}+\psi_{1}\right) \\
& \left.-\sin \left(\psi_{0}-\psi_{1}\right) \cos \left(\psi_{0}-\psi_{1}\right)\right\} / 2, \\
K_{3}=\left\{\tan \theta_{\mathrm{B}}\left[\cos ^{2}\left(\psi_{0}+\psi_{1}\right)-\cos ^{2}\left(\psi_{0}-\psi_{1}\right)\right]\right. & +\sin \left(\psi_{0}+\psi_{1}\right) \cos \left(\psi_{0}+\psi_{1}\right) \\
& \left.-\sin \left(\psi_{0}-\psi_{1}\right) \cos \left(\psi_{0}-\psi_{1}\right)\right\} / 2, \\
K_{4}=\left\{\tan \theta_{\mathrm{B}}\left[\sin ^{2}\left(\psi_{0}+\psi_{1}\right)-\sin ^{2}\left(\psi_{0}-\psi_{1}\right)\right]\right. & -\sin \left(\psi_{0}+\psi_{1}\right) \cos \left(\psi_{0}+\psi_{1}\right) \\
& \left.+\sin \left(\psi_{0}-\psi_{1}\right) \cos \left(\psi_{0}-\psi_{1}\right)\right\} / 2 .
\end{aligned}
$$

The terms with $\left(\psi_{0}+\psi_{1}\right)$ and $\left(\psi_{0}-\psi_{1}\right)$ in Eqs. (7) and ( 8 ) combine to make $K_{1}$ and $K_{2}$ insensitive to $\psi_{1}$ for small $\psi_{1}$, so that the strains obtained from Eq. ( $\left.1^{\prime}\right)$ are insensitive to small misorientations of the layer surface.

Equations (1)-(10) are applicable to $\left(h_{0} k_{0} l_{0}\right)$ rocking curves for which $\psi_{0}=0$. In this case the subscript $c$ refers to the rocking curve obtained upon reversing the diffraction vectors $\left(180^{\circ}\right.$ rotation of the sample about $\left.\left[h_{0} k_{0} l_{0}\right]\right)$. For $\psi_{1}$ less than $\sim 1^{\circ}, K_{2} \approx 0$ and $\epsilon_{3}$ is obtained directly using Eq. (5). The strain $\epsilon_{P}$ is then found using this $\epsilon_{3}$ in Eq. (5) together with the Bragg angle shifts for two equivalent planes.

\section{APPLICATION TO (001) SILICON ON (011̄2) SAPPHIRE}

An (0112) sapphire wafer with an (001) Si epilayer $\sim 4000 \AA$ thick was obtained from Union Carbide. The $\langle 100\rangle$ zone axes in the $\mathrm{Si}$, which have equivalent $\{440\}$ planes suitable for Bragg diffraction (using $\mathrm{CoK} \alpha_{1}$ radiation), were determined to be within $\sim 1^{\circ}$ of the layer surface. The $\langle 110\rangle$ zone axes nominally in the surface have equivalent $\{333\}$ planes suitable for Bragg diffraction (using $\mathrm{Fe} K \alpha_{1}$ ). Rocking curves were obtained from these planes and from (004) planes with $\mathrm{Fe} K \alpha_{1}$

TABLE I. X-ray parameters for the measured rocking curves (angles in degrees).

\begin{tabular}{ccrcccc}
\hline \hline $\begin{array}{c}\text { Reflecting } \\
\text { plane }\end{array}$ & Radiation & $\phi$ & $\theta_{B}$ & $\psi_{0}$ & $K_{1}$ & $K_{2}$ \\
\hline$(004)$ & $\mathrm{Fe} K \alpha_{1}$ & 0 & 43.27 & 0 & 1.0179 & 0 \\
$(004)$ & $\mathrm{Fe} K \alpha_{1}$ & 180 & 43.27 & 0 & 1.0179 & 0 \\
$(0 \overline{4} 4)$ & $\mathrm{Co} K \alpha_{1}$ & 135 & 68.71 & 45 & 1.7828 & 0.7828 \\
$(044)$ & $\mathrm{CoK} \alpha_{1}$ & 315 & 68.71 & 45 & 1.7828 & 0.7828 \\
$(404)$ & $\mathrm{CoK} \alpha_{1}$ & 45 & 68.71 & 45 & 1.7828 & 0.7828 \\
$(\overline{4} 04)$ & $\mathrm{CoK} \alpha_{1}$ & 225 & 68.71 & 45 & 1.7828 & 1.7828 \\
$(333)$ & $\mathrm{Fe} K \alpha_{1}$ & 0 & 67.92 & 54.74 & 1.2932 & 1.1721 \\
$(\overline{3} \overline{3} 3)$ & $\mathrm{Fe} K \alpha_{1}$ & 180 & 67.92 & 54.74 & 1.2932 & 1.1721 \\
$(3 \overline{3} 3)$ & $\mathrm{Fe} K \alpha_{1}$ & 90 & 67.92 & 54.74 & 1.2932 & 1.1721 \\
$(\overline{3} 33)$ & $\mathrm{Fe} K \alpha_{1}$ & 270 & 67.92 & 54.74 & & 1.2932 \\
\hline \hline
\end{tabular}


radiation. An $0.2 \mathrm{~mm}$ thick (001) silicon crystal $(1 \times 2$ $\mathrm{cm}$ ) was used as a reference crystal. A circular hole 5 $\mathrm{mm}$ in diameter was cut in the face of the reference crystal in order to permit $\mathrm{x}$ rays to simultaneously diffract from the silicon epilayer and the edge of the hole of the reference crystal (while the reference crystal was held on the Si layer by a viscous grease). The crystal axes of the reference crystal and the layer were aligned to within $1^{\circ}$. The sapphire wafer was held on a diffractometer stage, which permitted $360^{\circ}$ angular adjustment about the center of the hole in the reference crystal ([001] rotation axis in the layer with rotation angle $=\phi$ ).

The relevant parameters for the rocking curves obtained here are given in Table I. An (001) GaAs first crystal was used and a (004) reflection was selected for both x-ray wavelengths employed. The angles $\psi_{0}-\theta_{\mathrm{B}}$ and $\psi_{0}+\theta_{\mathrm{B}}$ are the nominal angles between the diffraction vectors and the surface of the silicon layer. Note that the $\{333\}$ reflections are more sensitive to $\epsilon_{P}$ (larger $K_{2}$ ) than the $\{044\}$ reflections. Here $K_{1}$ and $K_{2}$ vary by less than $0.1 \%$ with $-5^{\circ} \leqslant \psi_{1} \leqslant 5^{\circ}$ for the asymmetric reflections $\left(\psi_{1}\right.$ was measured to be less than $\left.1^{\circ}\right)$.

A superimposed pair of rocking curves from (004) reflections $\left(\phi=0^{\circ}\right.$ and $\left.180^{\circ}\right)$ is shown in Fig. 2. Peak positions were determined by least-squares fitting of a Gaussian to the curves over the range above approximately the half maximum of the peaks. The standard errors in the least-squares fits were used to obtain the the reported errors in the peak shifts. The primary source of error came from the fit to the relatively broad peaks

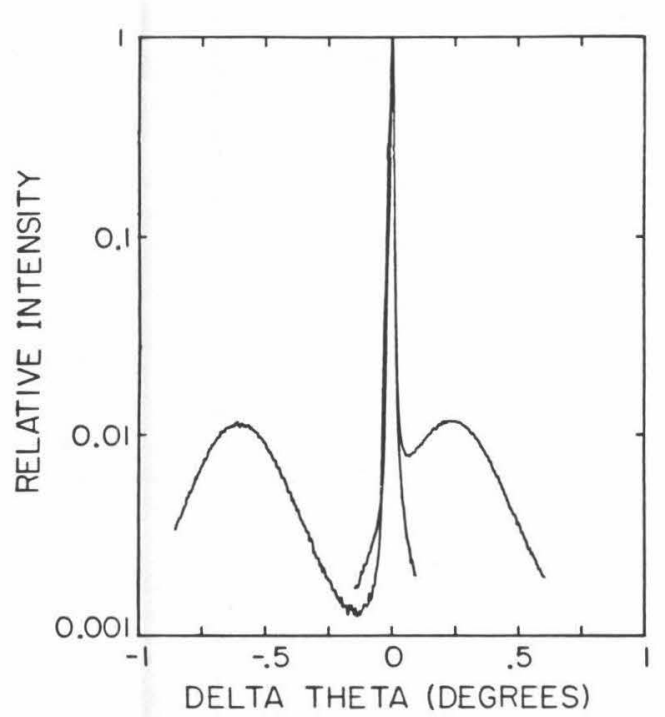

FIG. 2. Relative intensity of (004) reflections using $\mathrm{Fe} K \alpha_{1}$ from the silicon layer (broad peaks) and the reference crystal (sharp peaks). The curve showing the layer peak on the left was obtained with $\phi=0^{\circ}$ and the curve showing the layer peak on the right was obtained for $\phi=180^{\circ}$.
TABLE II. Measured Bragg peak shifts (degrees) and calculated strains.

\begin{tabular}{rcccc}
\hline \hline$\phi$ & $(h k l)$ & Peak shift & Calculated strain $(\%)$ & Strain direction \\
\hline 0 & 004 & $-0.597 \pm 0.011$ & $0.303 \pm 0.015$ & {$[001]$} \\
180 & 004 & $0.232 \pm 0.007$ & & \\
135 & $0 \overline{4} 4$ & $1.038 \pm 0.017$ & $-0.472 \pm 0.061$ & {$[100]$} \\
315 & 044 & $-1.203 \pm 0.014$ & & \\
45 & 404 & $0.512 \pm 0.010$ & $-0.531 \pm 0.071$ & {$[010]$} \\
225 & $\overline{4} 04$ & $-0.730 \pm 0.013$ & & \\
0 & 3233 & $-0.332 \pm 0.013$ & $-0.447 \pm 0.037$ & {$[110]$} \\
180 & $\overline{3} \overline{3} 3$ & $0.453 \pm 0.008$ & & \\
90 & $3 \overline{3} 3$ & $-0.348 \pm 0.012$ & $-0.425 \pm 0.032$ & {$[110]$} \\
270 & $\overline{3} 33$ & $0.499 \pm 0.016$ & & \\
\hline \hline
\end{tabular}

from the epilayer (diffractometer resolution was $10^{-4}$ degrees).

Table II lists the measured peak shifts, standard errors, and the strains calculated from these measurements. The [001] strain $\left\{\epsilon_{3}\right\}$ was calculated from the peak shifts from (004) reflections with $\phi=0^{\circ}$ and $180^{\circ}$. This strain was used in Eq. (5) to find $\epsilon_{P}$ with peak shifts from equivalent reflections.

\section{DISCUSSION}

It is possible to distinguish between rocking curve broadening due to particle size effects and distortion ef-

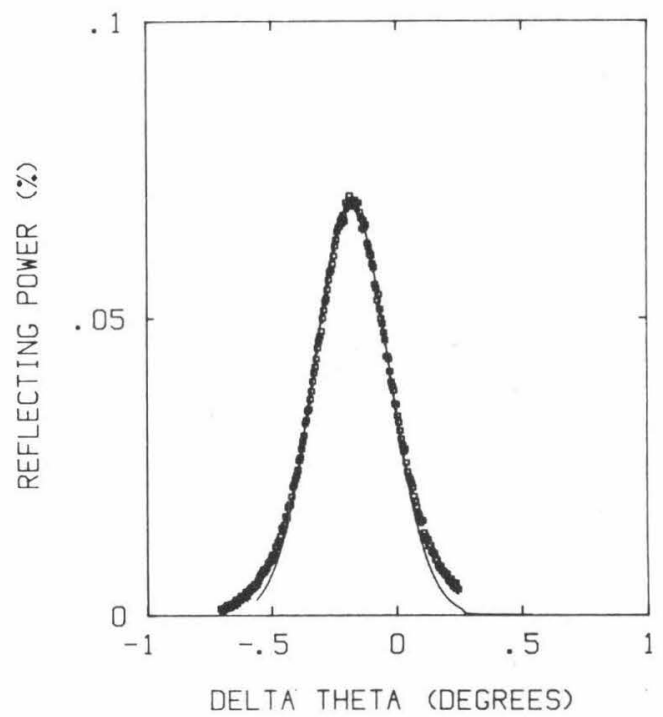

FIG. 3. The (004) rocking curves from an (001) silicon epilayer on $(01 \overline{1} 2)$ sapphire using FeK $\alpha_{1}$. The solid line is calculated using the kinematical theory for a $3900 \AA$ thick layer with constant $\epsilon_{3}=0.303 \%$. The calculated curve was broadened by convolution with a Gaussian ( 530 arcsec standard deviation). 
TABLE III. Calculated [001] strain using measured strains parallel to the layer, and calculated principal stresses.

\begin{tabular}{cc}
\hline \hline Measured strain directions & Calculated $\epsilon_{3}(\%)$ \\
{$[100]$ and $[010]$} & $0.369 \pm 0.050$ \\
{$[\overline{1} 10]$ and $[110]$} & $0.336 \pm 0.027$ \\
\hline Principal stresses calculated from principal strains \\
Direction & Stress, GPa \\
\hline$[100]$ & $-0.92 \pm 0.16$ \\
{$[010]$} & $-0.98 \pm 0.17$ \\
\hline \hline
\end{tabular}

fects. ${ }^{6,7}$ Particle size broadening arises from dislocation substructure and from discrete crystal "blocks" that are separated by twins. Deformation broadening is due to randomly distributed dislocations and to the strains caused by the twins. Particle size broadening alone produces a Lorentzian curve while deformation broadening produces a Gaussian. The silicon epilayer peak should exhibit both types of broadening since substructure in silicon epilayers on sapphire was observed by Dumin, ${ }^{1}$ and twins were observed by Abrahams and Buiocchi. ${ }^{8}$

The diffraction peaks from the silicon epilayer are considerably broadened, as compared to peaks calculated for a strained, defect-free layer. Figure 3 shows the (004) data obtained from the epilayer and a calculated rocking curve from a single (001) layer, $3900 \AA$ thick. ${ }^{9}$ The calculated curve for the layer was convoluted with a Gaussian (standard deviation $=530$ arcsec) to match the amplitude of the experimental rocking curve. The data show more prominent tails than the Gaussian convoluted curve indicating that some of the broadening results from particle size broadening due to dislocation substructure and twinning. Lattice distortion (from dislocations and twins) contributes a significant Gaussian component of broadening. The relatively large broadening of the layer peaks was the main source of uncertainty in the strains given in Table II.

The $\epsilon_{3}$ strain in the epilayer may be calculated from the measured $\epsilon_{P}$ strains along orthogonal axes in the layer using the known elastic coefficients for Si. Table III lists the calculated values of $\epsilon_{3}$, which agree with the measured value (Table II) within the uncertainty range using the standard errors of measurement. The strains in the [100] and [010] directions are principal strains ${ }^{2}$ so the strains in $[\overline{1} 10]$ and [110] directions are equal. The measured [ $[\overline{1} 10]$ and [110] strains are equal within the standard errors of measurement. Principal stresses, calculated from $\langle 100\rangle$ strains are also given in Table III. The stresses agree within the range of standard error with those calculated by Hughes for thermal stresses due to the differential thermal contraction between the silicon and sapphire upon cooling from $1100{ }^{\circ} \mathrm{C}$, and confirm his view that "the room-temperature stress in $\mathrm{Si} / \mathrm{Al}_{2} \mathrm{O}_{3}$ films is predominantly due to thermal expansion mismatch rather than to lattice-constant mismatch or other growth stresses."2

\section{CONCLUSIONS}

(1) A technique for measurement of the strain tensor in thin epitaxial layers has been developed and applied to Si on sapphire.

(2) The measured strain perpendicular to the layer, $0.303 \% \pm 0.015 \%$, was found to agree within experimental uncertainty with the perpendicular strain calculated from measured strains in the plane of the layer.

(3) The principal stresses, calculated from the measured strains, agree within experimental uncertainty with the thermal stresses developed upon cooling a coherent $\mathrm{Si}$ layer on sapphire from the growth temperature.

\section{ACKNOWLEDGMENTS}

Professor R. W. Cahn kindly provided constructive comments on the manuscript.

This work was supported by the National Science Foundation, under the Caltech Materials Research Group Grant No. DMR84-21119.

\section{REFERENCES}

'D. J. Dumin, J. Appl. Phys. 36, 2700 (1965).

${ }^{2}$ A. J. Hughes, J. Appl. Phys. 46, 2849 (1975).

${ }^{3}$ U. Bonse and I. Hartmann, Z. Kristallogr. 156, 265 (1981).

${ }^{4}$ U. Bonse and Z. Physik 153, 278 (1958).

${ }^{5}$ V. S. Speriosu and T. Vreeland, Jr., J. Appl. Phys. 56, 1591 (1984).

${ }^{6}$ M. Wilkens, Phys. Stat. Solidi A 2, 359 (1970).

${ }^{7}$ B. E. Warren, X-ray Diffraction (Addison-Wesley, Reading, MA, 1969).

${ }^{8}$ M. S. Abrahams and C. J. Buiocchi, Appl. Phys. Lett. 27, 325 (1975).

${ }^{9}$ V. S. Speriosu, J. Appl. Phys. 52, 6094 (1981). 